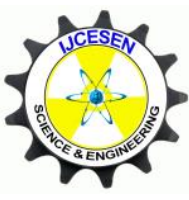

Copyright (C) IJCESEN
International Journal of Computational and

Experimental $\boldsymbol{S}$ cience and $\boldsymbol{E N g i n e e r i n g}$

(IJCESEN)

Vol. 6-No.2 (2020) pp. 98-101

http://dergipark.org.tr/en/pub/ijcesen

Research Article

\title{
Thermodynamics of the Quark Gluon Plasma in the Early Universe
}

\author{
Hamid ALZAKI*
}

Suleyman Demirel University, Science and Arts Faculty, Physics Department, 32200, Isparta-Turkey

* Corresponding Author : alshamary632000@yahoo.com

ORCID: 0000-0002-1142-8614

\section{$\underline{\text { Article Info: }}$}

DOI: $10.22399 /$ ijcesen.686962

Received : 09 February 2020

Accepted : 11 June 2020

\section{Keywords:}

Quark gluon plasma

Early Universe

Equation of states

\begin{abstract}
$\underline{\text { Abstract: }}$
The recent progress in collider experiments provides us with valuable information to understand the properties of the quark-gluon plasma. In this study, we provide the analytical solution to the differential equation of the time evolution of the temperature in the early Universe in the MIT bag model for gluons only. We discuss the equation of state of a QGP in a particular model, the possible signs for a plasma created in heavy-ion collisions and provide analytic solutions describing the time evolution of the energy density, temperature and pressure of this plasma in the early universe.
\end{abstract}

\section{Introduction}

The early Universe during the first 10 microsecond after the big bang was filled with soup of quarks and gluons, in other word Quark gluon plasma is a state of matter with the highest energy level in which the elementary particles that make up the hadrons of baryonic matter are free and are not affected by strong interactions between them under extremely high energy densities (with temperature higher than $150 \mathrm{MeV}$ ) [1]. Quarks, the subatomic particles carrying a fractional electric charge and one of two basic components of matter in the theory of standard model of particle physics. Gluons, the massless particles that "carry" the force between quarks, they are the particles that quarks exchange as they interact, or, in the language of modern physics, they mediate the strong force between quarks, that compose baryonic matter [2]. The conception, of the state of the quark gluon plasma have been improved and evolved by experimentally and theoretically studies. Experimentally by using colliders, through the relativistic heavy-ion collision experiments at Brook-haven National Laboratory in the United States of America and European Nuclear Council, Geneva in Switzerland. Theoretically, from the numerical simulation of finite temperature quantum chromo-dynamics on a lattice [3]. The new results obtained by som work about the nature of the QGP motivate them to achieve their logical results in the early Universe. This could be done through solving the Fried-mann equations, which allow them to determine the exact temporal development of thermodynamic quantities at the beginning of the universe. So we see that the study of quark matter is very important to understand the nature of universe which gave me an incentive to research in this field. The Quark Gluon Plasma was first proposed by Collins and Perry and others in the 1970s. Their arguments are based on the principle of asymptotic freedom in Quantum dynamics (QCD) is the widely accepted theory in the world that describing the strong interactions of quarks, gluons and nucleons [4]. It is the strongest comparison with other interactions and for example, the gravitational force is $\sim 10^{-39}$, the weak interaction is $\sim 1 / 137$ and the strong interaction is $\sim 1$. It is known through studies in the field of cosmology, the Universe is homogeneous and isotropic. Such characteristics indicate that the Fried-mann Robertson-Walker metric will parameterize the space-time, which has been introduced into the Einstein equations to provide the Fried-mann equations. So, of these latter equations, the time evolution equatio $\mathrm{n}$ can be derived in the following [5].

$\frac{d \varepsilon}{3 \sqrt{\varepsilon}(\varepsilon+B)}=-\sqrt{\frac{8 \pi G}{3}} d t$ 
It helps us to evaluate the temporal evolution of the density of energy $\varepsilon$, by we know just the pressure $\mathrm{P}$, $P \equiv P(\varepsilon)$. Throughout this study we analytically solved the above equation, using some previously inferred state equations and estimating the time evolution of certain thermodynamic quantities for gluon in the early universe, such as energy density $\varepsilon$, temperature $\mathrm{T}$ and pressure $\mathrm{P}$.

\section{Equation of State}

The equation of state (EOS), in thermodynamic, describes the relationship between the state functions of a thermodynamic system. It can describe the state of the system and the way it changes. Equations of state are used to identify the properties of gases, liquids and mixtures of liquids as well as the properties of solid matter. The system in thermodynamics (which is a department of physics and chemistry and a number of branches of engineering). A system of limited size (gas, liquid, solids, or a mixture there of). We conduct our observations on it, it is characterized by a balanced energy condition. This applies to a closed system, i.e. there is no heat or radiation exchange or material exchange between the system and the surrounding medium. This is in contrast to an "open system" and is in exchange with the surrounding medium, and between them there is an exchange of materials or heat [6]. In this study, the focus is on the equations of state (EOS) of the quark gluon plasma QGP and finding an analytical solution to a specific case in the case of the free gluons. Equations of state of the quark gluon plasma were extensively used in cosmology and astrophysics, where the first equation was derived in the MIT bag model [7]. We want to investigate how fluctuations in the EOS influence the time evolution of the gluons in the early universe. We believe this study is important in order to confirm numerical solution previously calculated by other researchers in order to be able to compare and think about other solutions for scientific research service.

\section{MIT Bag Model}

The MIT bag model is a very simple phenomenological model. It was developed by a physicist Chodos in 1974 at the Massachusetts Institute of Technology in Cambridge in the United States of America after a brief period wording of quantum chromo-dynamic and it became a main instrument for researchers in the field of hadron physics. Scientists evidenced that bag model results in to energy momentum conservation violation at the bag surface, unless the internal pressure inside the bag is balanced by an external pressure. This led to some modification to the model, by using quarks carrying colour, they managed to partially destroy the real vacuum inside the bag. Quarks are subjected by a fixed external pressure, to move only in a specific spatial area, one perception is that of flexible bag allows to the quarks to move freely inside it in case of quark confinement and so long as we don't allow them to be isolated out. But if you try to take the quark out, the bag extends and resists. This combination of quarks and real vacuum made it possible to treat the physics of the interior of the bag by troubled quantum chromo-dynamics, this is called troubled vacuum inside the bag. This change in the model of the bag leads to a new boundary case. The necessity condition of pressure balance at the surface can be written as follows [8-9].

$-\left.\frac{1}{2} n^{\mu} \partial_{\mu}\left(\sum_{i} \bar{\psi}_{i} \psi_{i}\right)\right|_{S}=\mathcal{B}$,

where $B$ is the bag constant, $i$ runs over all quarks contained in the bag, $n^{\mu}$ is normal to the surface at given point, $\psi \mathrm{N}$ component Dirac field and transformation of $\psi$ represents as follow

$\psi(x) \rightarrow \bar{\psi}(x)=U(x) \psi(x)=e^{-i \tau \cdot \alpha(x)} \psi(x)$,

where $U(x)$ is the operator of the transformation, $\tau$ generators and $\alpha(x)$ is the parameters of the transformation.

As we mentioned, inside the "bag", the quarks and gluons freely move and the deconfined phase (which describes a system of these particles that have relative freedom of movement), can be created by compressing the bags against one another. And after that, the plasma constituents are free to move through large spatial areas at these high baryon numbers and energy densities. A high temperature increases the existence of quark-anti-quark pairs. Even under these conditions, the bag constant still represents some non-perturbative element. Energy density and pressure are given respectively by

$\varepsilon=\frac{37 \pi^{2}}{30} T^{4}+\mathcal{B}$ and $p=\frac{37 \pi^{2}}{90} T^{4}-\mathcal{B}$.

Upon eliminating the temperature $T$ from the above equations we get

$p(\varepsilon)=\frac{1}{3}(\varepsilon-4 \mathcal{B})$.

In this study, the focus was on solving the time evolution of temperature in the early universe in MIT bag model for case of gluon only. The equation of state of gluon are given $[7,10]$

$\varepsilon_{g}=\frac{8 \pi^{2}}{15} T^{4}+B$ 
$p_{g}=\frac{8 \pi^{2}}{45} T^{4}-B$

Where $\varepsilon_{g}$ is the energy density of gluon, $p_{g}$ is the pressure of gluon, $\mathrm{T}$ is the temperature and $\square \square$ is the bag constant.

\section{Analytical Solution for Temperature}

In this section, we derive the analytical solution of differential equation that represents the time evolution of temperature. We start with the time evolution of energy density equation which is given as

$\frac{d \varepsilon}{3 \sqrt{\varepsilon}(\varepsilon+B)}=-\sqrt{\frac{8 \pi G}{3}} d t$

From the eqs [6,7] we obtained

$d \varepsilon=\frac{4 \times 8 \pi^{2} T^{3}}{15} d T,(\varepsilon+B)=\frac{32 \pi^{2}}{45} T^{4}$

(9)

By substitute Eq. 9 in Eq. 8 and integration of both sides we get

$\int_{T_{0}}^{T} \frac{1}{T \sqrt{\frac{8 \pi^{2}}{15} T^{4}+B}} d T=-\int_{t_{0}}^{t} \sqrt{\frac{8 \pi G}{3}} d t$

Let $x=T^{2} \Rightarrow d x=2 T d T, d T=\frac{d x}{2 \sqrt{x}}$. By substitute in above Eq. we get

$\frac{1}{2} \int_{\sqrt{x_{0}}}^{\sqrt{x}} \frac{1}{x \sqrt{\frac{8 \pi^{2}}{15} x^{2}+B}} d x=-\int_{t_{0}}^{t} \sqrt{\frac{8 \pi G}{3}} d t$

$\frac{\sqrt{15}}{4 \sqrt{2} \pi} \int_{\sqrt{x_{0}}}^{\sqrt{x}} \frac{1}{x \sqrt{x^{2}+\frac{15 B}{8 \pi^{2}}}} d x=-\int_{t_{0}}^{t} \sqrt{\frac{8 \pi G}{3}} d t$

$\frac{\sqrt{15}}{4 \sqrt{2} \pi} \int_{\sqrt{x_{0}}}^{\sqrt{x}} \frac{1}{x \sqrt{x^{2}+\alpha^{2}}} d x=-\int_{t_{0}}^{t} \sqrt{\frac{8 \pi G}{3}} d t$,

where $\alpha^{2}=\frac{15 B}{8 \pi^{2}}$

$-\frac{\sqrt{15}}{4 \alpha \sqrt{2} \pi}\left[\ln \left|\frac{\alpha+\sqrt{x^{2}+\alpha^{2}}}{x}\right|\right]_{\sqrt{x_{0}}}^{\sqrt{x}}=-\left[\sqrt{\frac{8 \pi G}{3}} t\right]_{t_{0}}^{t}$

$\ln \frac{T_{0}^{2}\left[\alpha+\sqrt{T^{4}+\alpha^{2}}\right]}{T^{2}\left[\alpha+\sqrt{T_{0}^{4}+\alpha^{2}}\right]}=\frac{K}{a}\left(t-t_{0}\right) ; a=\frac{\sqrt{15}}{4 \alpha \sqrt{2} \pi}, k=\sqrt{\frac{8 \pi G}{3}}$

$\frac{T_{0}^{2}\left[\alpha+\sqrt{T^{4}+\alpha^{2}}\right]}{T^{2}\left[\alpha+\sqrt{T_{0}^{4}+\alpha^{2}}\right]}=e^{\frac{K}{a}\left(t-t_{0}\right)}$

$\frac{T^{2}\left[\alpha+\sqrt{T_{0}^{4}+\alpha^{2}}\right]}{T_{0}^{2}\left[\alpha+\sqrt{T^{4}+\alpha^{2}}\right]}=e^{-\frac{K}{a}\left(t-t_{0}\right)}$
$\frac{T^{2}}{\left[\alpha+\sqrt{T^{4}+\alpha^{2}}\right]}=C ; C=\frac{T_{0}^{2}}{\left[\alpha+\sqrt{T_{0}^{4}+\alpha^{2}}\right]} e^{-\frac{K}{a}\left(t-t_{0}\right)}$

$T^{2}=\frac{2 \alpha C}{1-C^{2}}$

$T=\sqrt{\frac{2 \alpha C}{1-C^{2}}}$

Below we show the graphic representation of the time evolution of temperature for gluon state only in this model

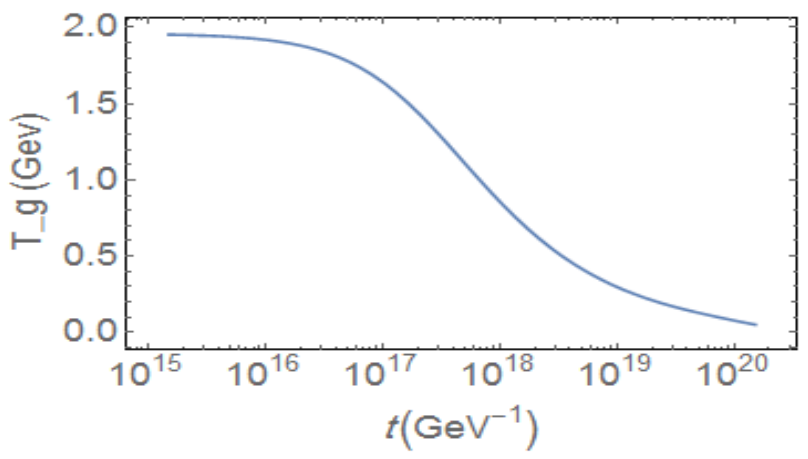

Figure 1. Time evolution of temperature.

In this solution we used the initial condition [11], it was converted to natural units, Giga-electron volts as follow

$$
\begin{aligned}
\varepsilon_{i}\left(t_{i}\right)= & 10^{7} \mathrm{MeV} / \mathrm{fm}^{3}=7.674 \times 10(\mathrm{Gev})^{4} \text { at } t_{i} \\
= & 10^{-9} \mathrm{~s}=1.52 \times 10^{15}(\mathrm{Gev})^{-1}
\end{aligned}
$$

In graphical representation. The evolution from the time of the electroweak transition phase $t_{i}=1.52 \times$ $10^{15}(\mathrm{Gev})^{-1}$, to the time of the quantum chromodynamics phase transition, $t_{f}=1.52 \times$ $10^{20}(\mathrm{Gev})^{-1}$. For the current study, we are mainly interested in obtaining analytic equations that describe the time evolution of the temperature, energy density and pressure for the plasma in the early universe during the time period discussed above. In figure 1 we display the time evolution of the temperature resulting by solving Eq. 1 with the MIT bag model equation of state of a gluon gas after converting it in terms of temperature and time. By looking at the figure, one can notice that the temperature was very high and then began to drop dramatically during this period. From the above equation of temperature we can find the energy density and pressure to same state respectively

$\varepsilon_{g}=\frac{8 \pi^{2}}{15}\left(\frac{2 \alpha C}{1-C^{2}}\right)^{2}+B$
$p_{g}=\frac{8 \pi^{2}}{45}\left(\frac{2 \alpha C}{1-C^{2}}\right)^{2}-B$ 


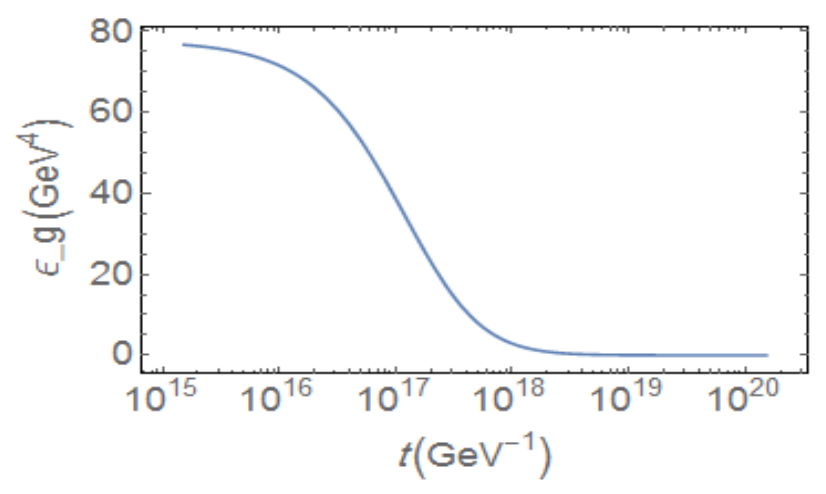

Figure 2. Time evolution of energy density.

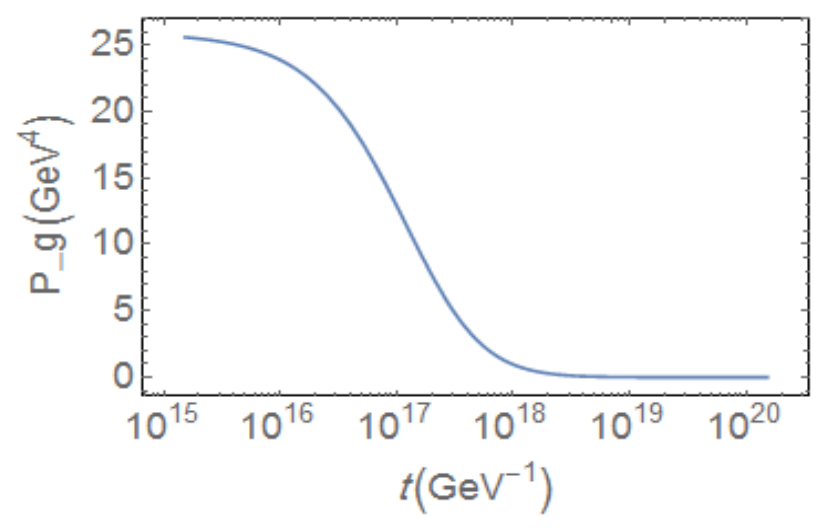

Figure 3. Time evolution of pressure.

In figures 2 and figure 3 we displayed time evolution of energy density and time evolution of pressure respectively.

\section{Conclusions}

As mentioned earlier in this work, in view of the experimental and theoretical studies that relevant to cosmology, show that the Universe was filled with dense plasma. This plasma consisted of deconfined quarks and gluons. This case of plasma is called as Quark-Gluon plasma. So, understanding this state allows us to learn more about the early universe. During the era of Quark-Gluon Plasma, the area of the relevant parameter consists of the total energy density, pressure, and temperature. Owing to the experimental data of heavy ion collisions, modern astrophysical measurements and also the developments in lattice QCD calculations, our understanding of the equation of state of the quark gluon plasma has been growing rapidly. These advanced results have helped us to understand of the time evolution of thermodynamic parameters at the beginning of the universe. We took the equations of state for the gluon that binds energy density and pressure to its temperature in the MIT bag model and solved Fried-man's differential equations that govern the time evolution of energy density, temperature, and pressure. We have showed that changes in the equations of state of gluon can lead to changes in the time evolution of these thermodynamic parameters in the early Universe in MIT bag model. Finally, we confirm that there are still some other components of the quark gluon plasma phase and other analytical solution for various models.

\section{Acknowledgement}

Author thanks to Dr. Gaber Faisal for his guidance during preparation and publication of this paper.

\section{References}

[1] Rafelski, Johann and Berndt Muller. Strangeness production in the quark gluon plasma. Physical Review Letters 48 no. 16 (1982): 1066.

[2] Markum, H., M. Meinhart, G. Eder, M. Faber, and H. Leeb. "Interactions between quark clusters in lattice QCD." Physical Review D 31, no. 8 (1985): 2029.

[3] Pickering, Andrew, and James T. Cushing. "Constructing quarks: A sociological history of particle physics." (1986): 381-383..

[4] Griffiths, David. Introduction to elementary particles. John Wiley \& Sons, 2008.

[5] Collins, John C., and Malcolm J. Perry. "Superdense matter: neutrons or asymptotically free quarks?.” Physical Review Letters 34, no. 21 (1975): 1353.

[6]http://en.wikipedia.org/wiki/Equation of state 2011 $/ 11 / 2$

[7] Chodos, Ao, R. L. Jaffe, K. Johnson, Charles B. Thorn, and V. F. Weisskopf. "New extended model of hadrons." Physical Review D 9, no. 12 (1974): 3471.

[8] Guardo, G. L., V. Greco, and M. Ruggieri. "Energy density fluctuations in Early Universe." In AIP Conference Proceedings, vol. 1595, no. 1, pp. 224227. American Institute of Physics, 2014.

[9] Florkowski, Wojciech. "The realistic QCD equation of state in relativistic heavy-ion collisions and the early Universe." Nuclear Physics A 853, no. 1 (2011): 173 188.

[10] Sanches Jr, S. M., F. S. Navarra, and D. A. Fogaça. "The quark gluon plasma equation of state and the expansion of the early Universe." Nuclear Physics A 937 (2015): 1-16.

[11] Fogaça, D. A., L. G. Ferreira Filho, and F. S. Navarra. "Nonlinear waves in a quark gluon plasma." Physical Review C 81, no. 5 (2010): 055211. 